\title{
Digital Spectrometric System for Characterization of Mixed Neutron - Gamma Field in the Experimental Reactor LR-0
}

\author{
Filip Mravec ${ }^{1, \mathrm{a}}$, Zdenek Matej $^{1}$, Frantisek Cvachovec ${ }^{2}$, Michal Kostal $^{3}$, Martin Veskrna ${ }^{1}$, \\ and Vaclav Prenosil ${ }^{1}$ \\ ${ }^{1}$ Masaryk University, Faculty of Informatics, Botanicka 68a, 60200 Brno, Czech Republic \\ ${ }^{2}$ University of Defence, Kounicova 65, 66210 Brno, Czech Republic \\ ${ }^{3}$ Research Center Rez ltd., 25068 Husinec-Rez 130, Czech Republic
}

\begin{abstract}
LR-0 reactor is an experimental reactor in NRI Rez, Czech Republic. So far an analog apparatus was used for measurements of the space-energy distribution of the neutron gamma mixed field inside the reactor vessel. Recently we measured in LR-0 with fully digital apparatus using Agilent digitizer and compared our results with older established results from analog apparatus and also with MCNP calculations.
\end{abstract}

\section{Introduction}

In the past an analogue spectrometer was used for neutron and gamma spectra measurements [1]. Analog electronics led to low rates of processed pulses and as a result exposure time had to be significantly prolonged. This disadvantage can be overcome by digital processing of pulses from neutron detector. Another advantage of digital approach is the reduction of weight and size of the spectrometric apparatus.

Second chapter describes a setup of a reactor vessel where experiments took place as well as measurement apparatus and lists methods used to process data and calculate spectra. Third chapter describes and comments on results obtained from performed experiments.

\section{Experimental Set-up}

The zero-power LR-0 reactor was designed for research on neutron - physical parameters of cores of the pressurized water reactor. The core was primarily intended for static measurements of reactivity worth. This can be performed by inserting the studied object into the core and measuring the rise in water level that is allowed by the specific construction of LR-0 reactor. This core consists of well characterized materials and has precise geometrical description of its components and thus can be also used to calibrate dosimetry devices for neutron dosimetry because for such purposes the neutron spectra must be precisely defined.

\footnotetext{
${ }^{a}$ Corresponding author: xmravec@fi.muni.cz
}

This is an Open Access article distributed under the terms of the Creative Commons Attribution License 2.0, which permits unrestricted use, distribution, and reproduction in any medium, provided the original work is properly cited. 

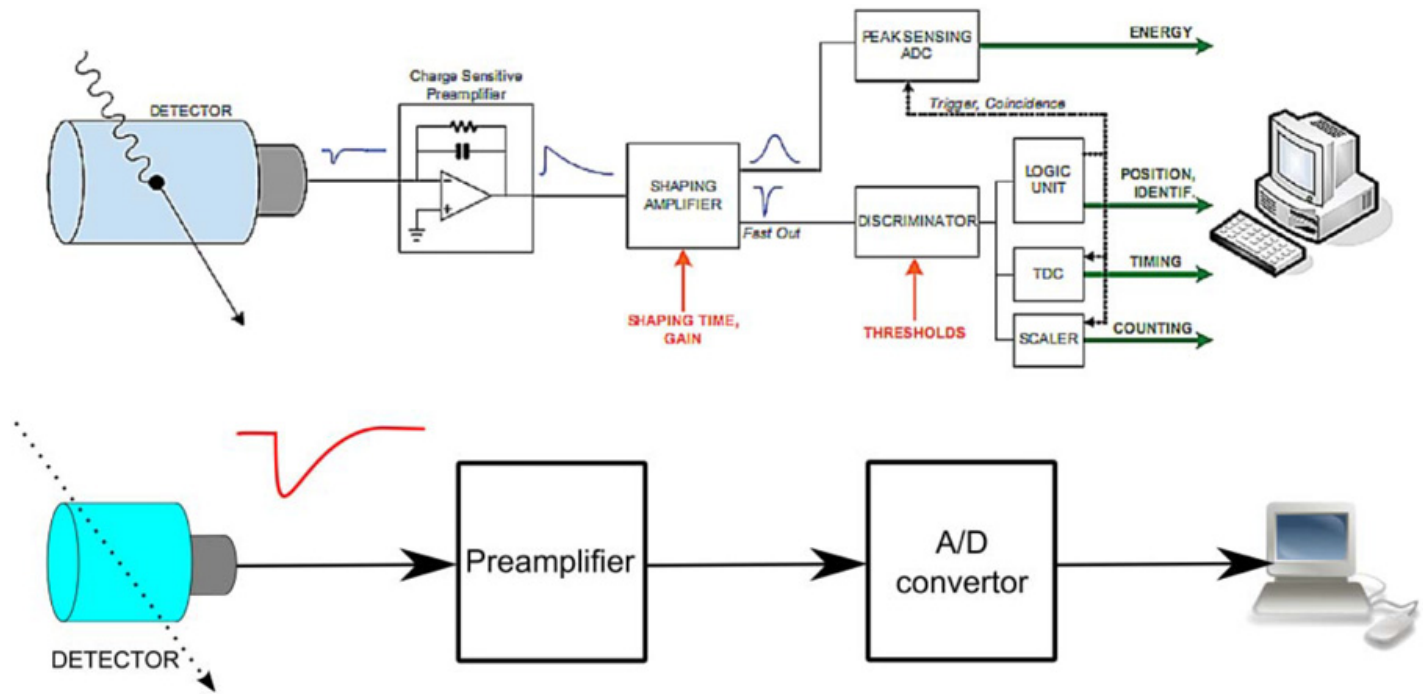

Figure 1. Difference between analog (up) and digital apparatus (down).

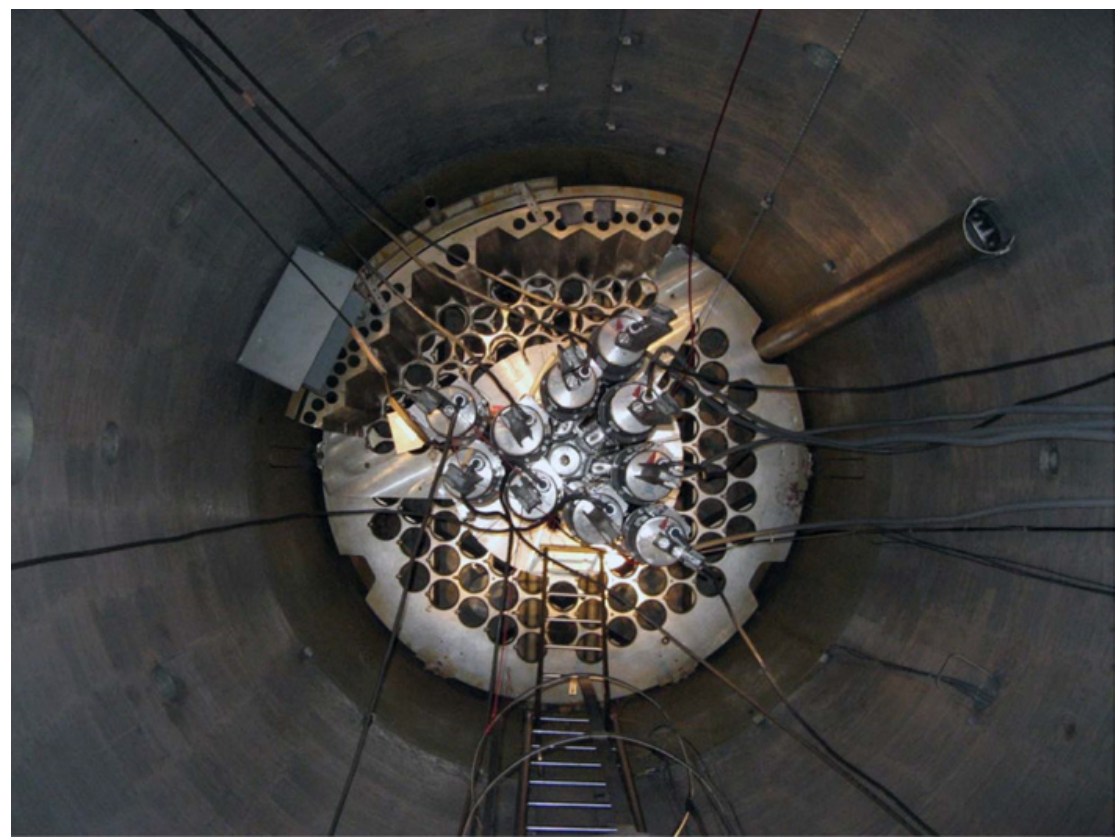

Figure 2. Setup inside the LR-0 vessel.

Applications mentioned above require the knowledge of space-energy distribution of mixed neutrongamma field. Fast neutron spectrometry in LR-0 was covered by analog spectrometer in the past. Our goal is to replace this apparatus with two-parametric digital spectrometers [2].

Figure 2 shows the inside of LR-0 reactor vessel. Active zone arrangement of LR-0 is shown in Fig. 3. Active zone in this setup consists of nine fuel cartridges. This zone is placed in the center of reactor vessel. 


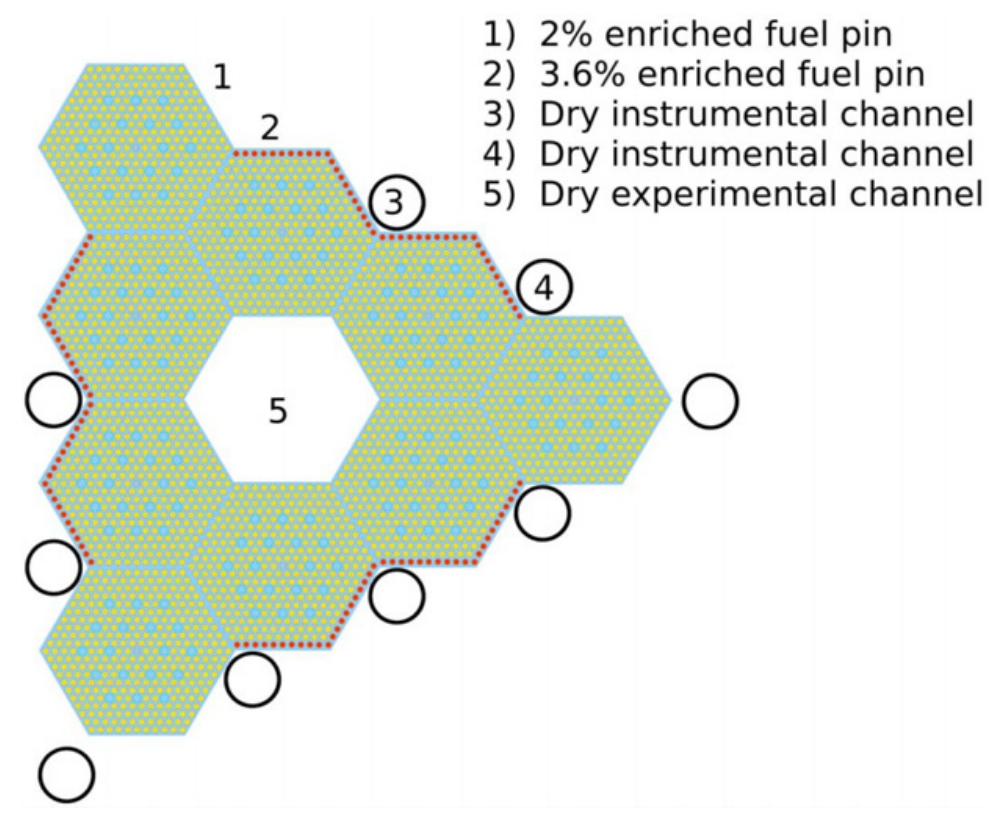

Figure 3. Active zone arrangement.

Three positions were selected and used for measurements. Measurement position 1 is situated in the middle of the active zone, i.e. position number 5 in Fig. 3. Positions 2 and 3 were outside of the active zone. Position 2 is placed next to the external wall of the active zone, i.e. position number 2 in Fig. 3. Last measurement position - position 3 - was situated 0.5 meter from position 2 away from active zone. Measurement position consisted of dry experimental channel. A detector is placed in the center of this dry channel.

Two parametric stilbene detectors were used for measurements. A cylindrical stilbene crystal (dimensions of $10 \times 10 \mathrm{~mm}$ ) was used for the measurement of neutron spectra in the first position. For measurement of gamma spectra $45 \times 45 \mathrm{~mm}$ stilbene crystal was used due to its better gamma detection efficiency. Gamma spectra were measured in all three positions.

U1066A Acqiris 12-bit digitizer was used for pulse digitization with sampling frequency of $420 \mathrm{MS} / \mathrm{s}$. This digitizer has two programmable standard signal input channels, each with voltage ranging from $250 \mathrm{mV}$ to $10 \mathrm{~V}$.

A well-established method based on Charge Comparison was used for separation of pulses from neutron or gamma induced pulses. To further improve this separation a modification of this algorithm was made to eliminate variations of zero level in the signal due to the length of the cable between detector and digitizer. This modification means that for each pulse first four samples were used to determine the actual zero value. This zero value was then subtracted from all the pulse samples.

Energy calibration was performed with ${ }^{137} \mathrm{Cs}$ and ${ }^{60} \mathrm{Co}$ sources for gamma detection and moreover using detector light output function for neutrons. The deconvolution of neutron spectra was done with the Expectation Maximization algorithm. Derivation of EM algorithm can be found in [3]. The EM algorithm is an iterative convergent algorithm that produces non-negative results given non-negative detector response function and apparatus spectrum (obtained from neutron pulses).

Figure 1 contains two schemas describing the older analog apparatus and the new digital one. Pulse separation is done as a part of analog processing within the analog apparatus contrary to digital approach. The rest of processing is the same for both approaches. 


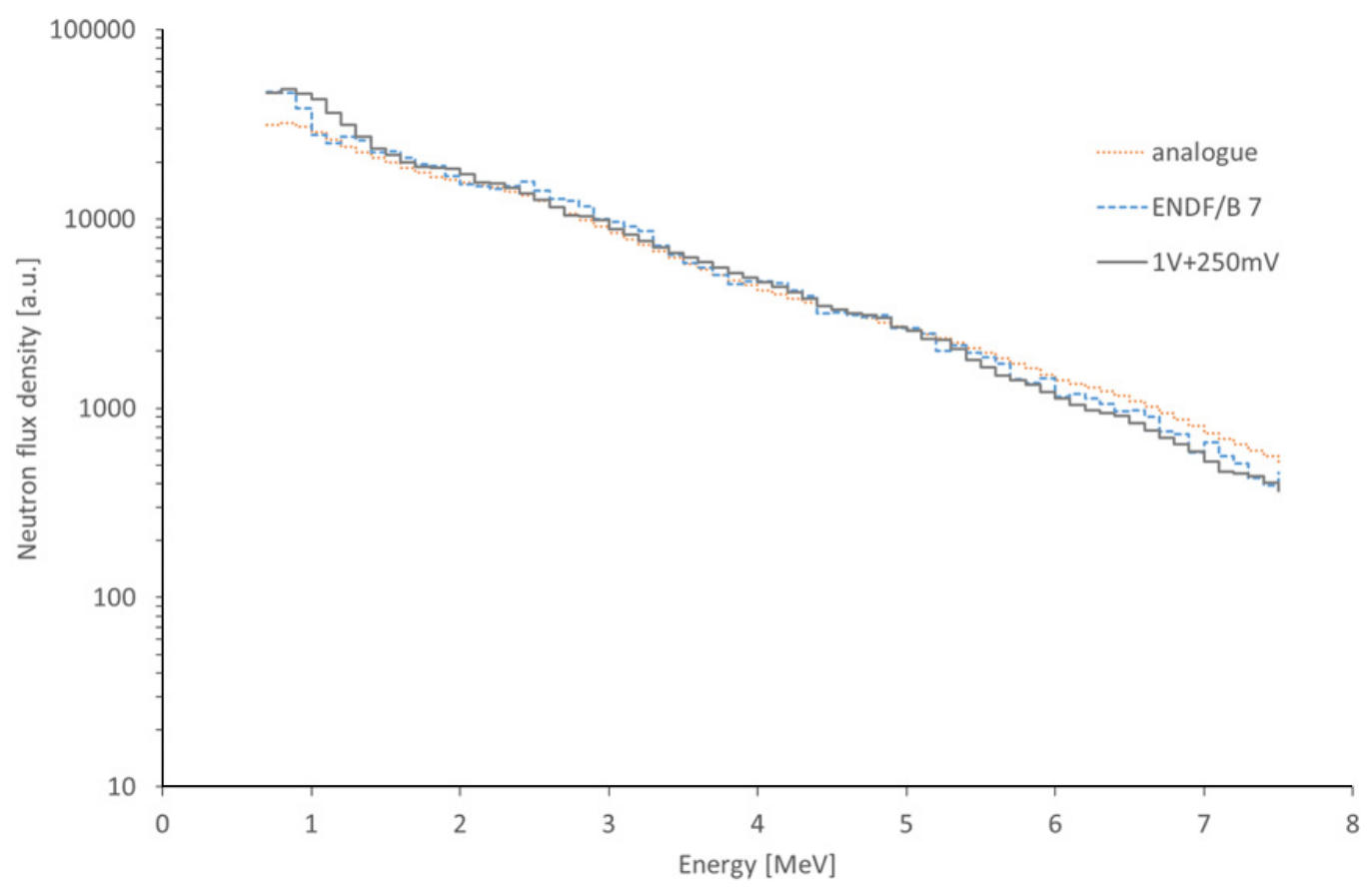

Figure 4. Neutron spectrum measured with 2 channels compared to analog measurement and calculation.

\section{Results}

Figures 4 and 5 depict comparisons of spectra calculated from data obtained by digital apparatus and spectra obtained from analog spectrometer. Additionally spectra calculated by Monte Carlo simulation with MCNPX 2.6.0 code [4] using ENDF/B VII nuclear data [5] are included.

Figure 4 shows a spectrum obtained from 2 input channels of the digitizer, one with input voltage of $1 \mathrm{~V}$ and the other with $250 \mathrm{mV}$. Using both channels of digitizer each with different amplification of the signal helps to extend the energy range of registered particles. Acquisition time of this measurement was 419 seconds.

The spectrum shown in Fig. 5 is obtained from one channel with input voltage of $2 \mathrm{~V}$. Acquisition time was 700 seconds. These spectra agree quite well with both calculations and spectra from analog apparatus. Biggest discrepancy is in low energies, possibly due to insufficient pulse separation.

Figure 6 shows gamma spectra measured in positions 1 (central), 2 and 3 of reactor vessel with channel input voltage of $2 \mathrm{~V}$. Acquisition time of measurement for position 2 and 3 was $100 \mathrm{~s}$, for position 1 it was $419 \mathrm{~s}$, i.e. the same as for neutron spectra from Fig. 4. Shape of these spectra is influenced by several reactions inside the reactor vessel. A peak at the energy $0.76 \mathrm{MeV}$ is created by ${ }^{95} \mathrm{Zr}$ and a peak at the energy of $2.23 \mathrm{MeV}$ by hydrogen. There are also peaks created by ${ }^{56} \mathrm{Fe}$ at energies $5.9 \mathrm{MeV}$ and $7.8 \mathrm{MeV}$. A drop in gamma flux intensity can be seen as detector moves away from the center of active zone, i.e. from central position to position 3 .

While it is possible to measure and calculate neutron spectra in the central channel where neutron pulses constituted about $10 \%$ of all detected pulses, in position 2 this ratio dropped to $2 \%$ and in position 3 to only $0.05 \%$. These low ratios together with limited exposure time made it impossible to calculate reliable neutron spectra in positions 2 and 3 .

To evaluate the uncertainties of computed spectra from digital apparatus we used Monte Carlo method. Description of this method can be found in [3]. Relative errors obtained from this method were 
$15^{\text {th }}$ ISRD

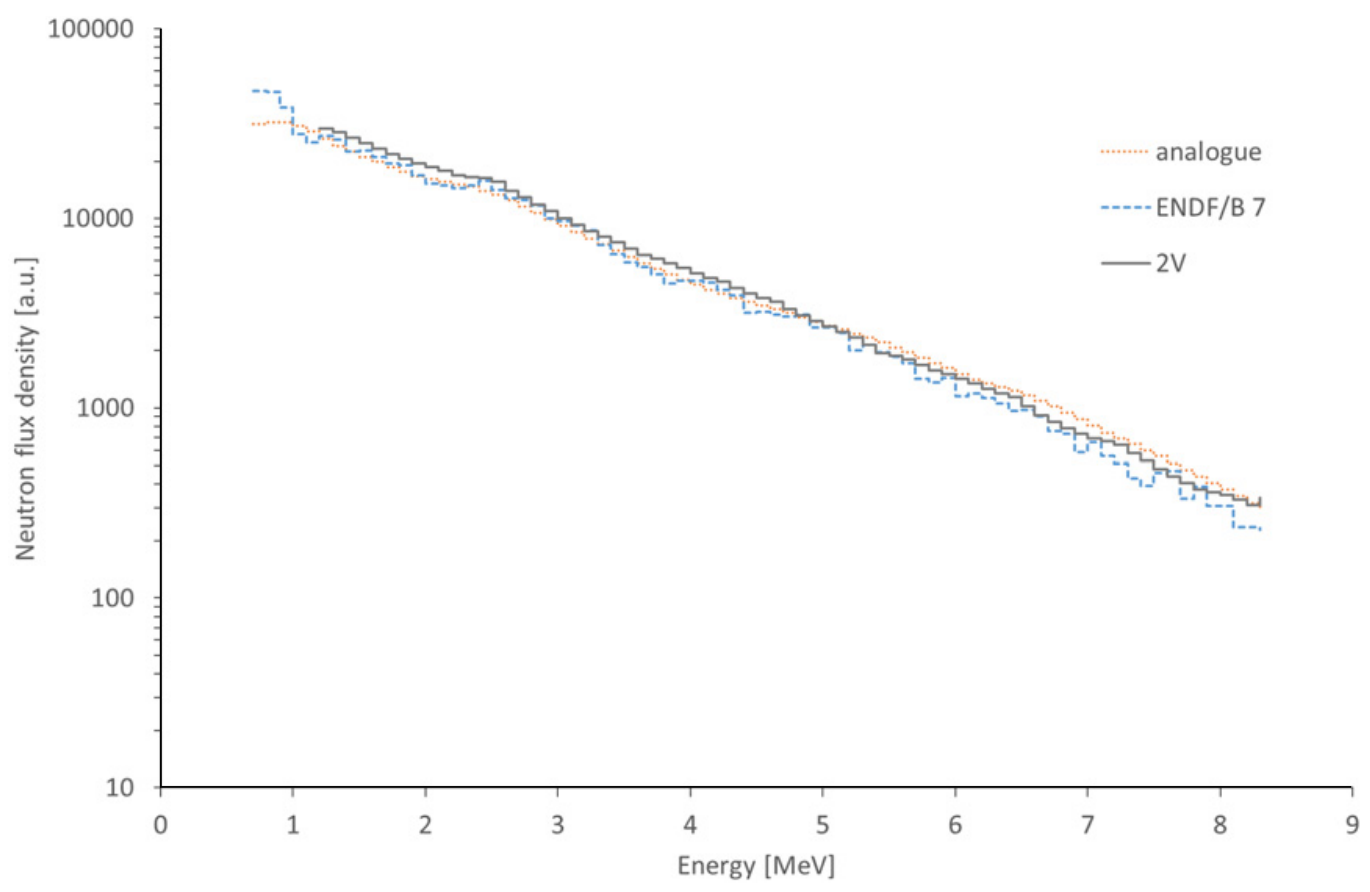

Figure 5. Neutron spectrum measured with 1 channel compared to analog measurement and MCNP calculation.

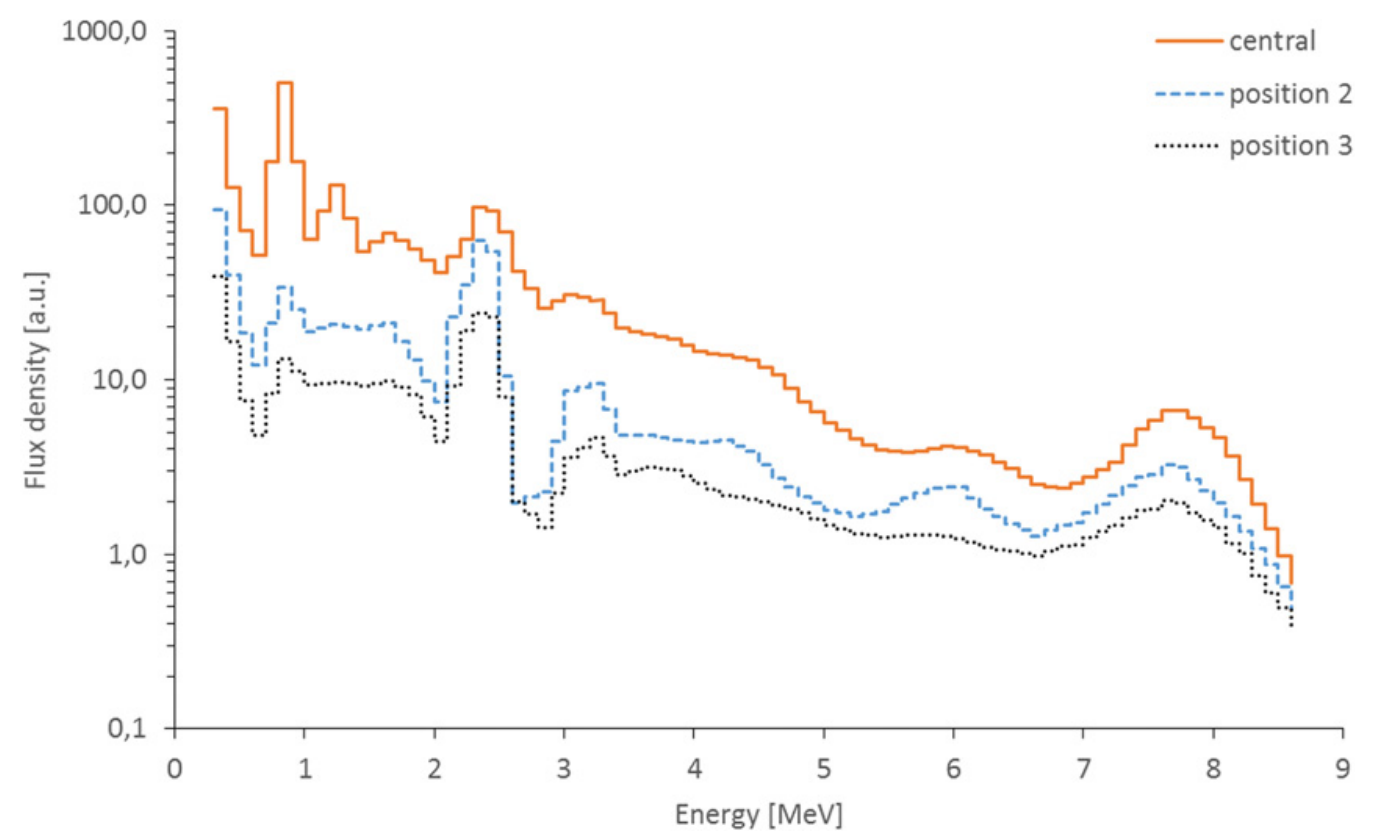

Figure 6. Gamma spectra measured in three different locations within the reactor vessel. 
of the order of magnitude of $10^{-2}$. Relative error for small energies (below $1 \mathrm{MeV}$ ) was approximately 0.01 and it increases for higher energies - it is about 0.07 for $7 \mathrm{MeV}$. Quantization error of the $\mathrm{AD}$ converter was close to $0.012 \%$ and quantization noise is close to $74.4 \mathrm{~dB}$. Global error of the implementation of the integration method - Midpoint Rectangle Approximation Method - was estimated using approximation by the Marrone equation of the detector response and is close to $0.0066 \%$.

\section{Conclusion}

From figures presented above it is reasonable to conclude that fully digital approach to neutron flux measurements inside the reactor LR-0 is possible. This approach leads to higher pulse rate and to wider range of processing possibilities which can furthermore improve measurement results. For example it is possible to move separation of pulses to a dedicated chip behind AD converters to speed up the pulse processing and to reduce the necessary bandwidth that PC must provide (from hundreds values per digitized pulse to only few values per pulse).

Even though the agreement between analog and digital processing and MCNP calculation is not ideal, these results suggest that with further improvements (e.g. better pulse separation and more precise calibration) it will be suitable for measurements of space-energy distributions in neutron-gamma mixed field in LR-0 and other facilities.

These experiments have been supported by Technology Agency of the Czech Republic (project TA01011383) and by project K215 - Support of mathematical and physical research, University of Defence, Brno.

\section{References}

[1] Z. Bures, J. Cvachovec, F. Cvachovec, P. Celeda, B. Osmera, Proc. Int. Conf. 11th Symposium on Reactor Dosimetry, Belgium, 194-201 (2002)

[2] Z. Matej, M. Amiri, F. Cvachovec, V. Prenosil, F. Mravec, Z. Kopecky, Progress in Nuclear Science and Technology, Japan (2012)

[3] J. Cvachovec, F. Cvachovec, Advances in Military Technology, 1, 5-28 (2008)

[4] D.B. Pellowitz, Los Alamos Report No. LA CP 02408 (2007)

[5] M.B. Chadwick, P. Oblozinsky, M. Herman, Nuclear Data Sheets, 107, 2931-3060 (2006) 